EUROPEAN JOURNAL OF PURE AND APPLIED MATHEMATICS

Vol. 11, No. 1, 2018, 315-330

ISSN 1307-5543 - www.ejpam.com

Published by New York Business Global

\title{
Regularization of heptahedra using geometric element transformation method
}

\author{
Santu Dey ${ }^{1}$, Budhhadev Pal ${ }^{2, *}$, Arindam Bhattacharyya ${ }^{1}$ \\ 1 Department of Mathematics, Jadavpur University, Kolkata-700032, India \\ 2 Institute of Science, Department of Mathematics, Banaras Hindu University, Varanasi, \\ Uttar Pradesh 221005, India
}

\begin{abstract}
By Geometric element transformation method (GETMe) always we get a new element. In this paper, we investigate the regularization of heptahedra using GETMe. Energy function is a cost function for heptahedra which is also applicable for octahedra, decahedra, hexahedra etc. is defined by a particular process, which we call base diagonal apex method (BDAMe). We also try to find the characterization of different cost function using BDAMe when we transform a heptahedra by GETMe.
\end{abstract}

2010 Mathematics Subject Classifications: 65M50, 51M04.

Key Words and Phrases: Heptahedron, mesh quality, iterative method, regularization, finite element mesh, cost functions.

\section{Introduction}

In many finite element applications unstructured tessellations of the geometry under consideration play a fundamental role. Therefore, the generation of quality meshes are essential steps of the simulation process, since mesh quality has an impact on solution accuracy and the efficiency of the computational methods involved [1, 2, 6, 9].

In [11], Geometric Element Transformation Method (GETMe) is represented as a simple geometric operation. The effect of this transformation, if applied iteratively, gives asymptotical but rapid regularization of the initial element. The authors of [11] have also introduced the concepts of global smoothing control and gave an algorithmic description of this method. The potential of the GETMe based smoothing is also illustrated there. In [13], the generalized transformation was presented and also described the enhanced GETMe-based smoothing by introducing the concepts and quality metric used for smoothing and termination control. Numerical results and a comparison to other smoothing methods, both geometrical and optimization-based were also given. In [12],

${ }^{*}$ Corresponding author.

Email addresses: santu.mathju@gmail.com (S. Dey), buddha.pal@rediffmail.com (B. Pal), bhattachar1968@yahoo.co.in (A. Bhattacharyya) 
the simultaneous GETMe smoothing algorithm based on transforming all mesh elements and the sequential GETMe algorithm based on transforming only the worst mesh element were described.

Basically, the geometric element transformation method (GETMe) is a geometry-based smoothing method for mixed and non-mixed meshes. It is based on a simple geometric transformation applicable to elements bounded by polygons with an arbitrary number of nodes. The transformation, if applied iteratively, leads to a regularization of the polygons. Global mesh smoothing is accomplished by averaging the new node positions obtained by local element transformations. Thereby, the choice of transformation parameters as well as averaging weights can be based on the element quality which leads to high quality results.

The most usual technique to improve the quality of a valid mesh, that is, one that does not have inverted elements, are based upon local smoothing. Usually, objective functions are appropriate to improve the quality of a valid mesh, but they do not work properly when there are inverted elements. To avoid this problem we can proceed as Freitag et al in $[3-5]$.

In this paper, we have defined the characterization of energy function of a heptahedra using base diagonal apex method (BDAMe). Also, we have defined the new apex transformation of heptahedron whose base points are fixed. Then we have discussed regularization properties of a heptahedra and tried to regularize by using geometric element transformation method (GETMe). Finally we have studied the characterization of energy function of a particular type of heptahedron using GETMe and BDAMe.

\section{Characterization of Energy Function of a Heptahedron}

For a 3-complex, the cost function referred as the energy function that discussed in [10]. But, we cannot estimate the energy function of all 3-D shapes. In [8], the chacterization of energy function of pentahedron have been defined. In this paper, we shall try to estimate the energy function of heptahedron by base diagonal apex method (BDAMe).

\subsection{Base Diagonal Apex Method(BDAMe):}

In [8], A. Bhattacharyya and B. Pal have defined base diagonal apex method. In this method, we add the two diagonal of the base of the pyramid and then add between the intersection point of the diagonal and the apex of the pyramid. This line (from apex to the intersection point of the diagonals) may be the height of the pyramid or may not be the height of the pyramid, totally depend upon the type of pyramid we choose. If we follow this method, we get six 3-simplex, that is, six tetrahedra. Now, each tetrahedron has a cost function or energy function. Therefore, we get six cost functions and then we can easily define cost function for heptahedra and to define the cost function of 3-D shapes except 3 -simplex, we introduce the function $h\left(v_{i}, \sigma^{n}\right)$, the signed distance from $c\left(\sigma^{n}\right)$ to aff $\left(\sigma_{i}^{n-1}\right)$ with convention that $h\left(v_{i}, \sigma^{n}\right) \geq 0$ when $c\left(\sigma^{n}\right)$ and $v_{i}$ are the same side of aff $\left(\sigma_{i}^{n-1}\right)$. Here $\sigma^{n}$ are the $n$-simplex, $c\left(\sigma^{n}\right)$ the circumcenter of the $n$-simplex, facet $\operatorname{aff}\left(\sigma_{i}^{n-1}\right)$ and vertex $v_{i}$. We have worked on all 3-D figures, so in that case $n=3$. The magnitude of $h\left(v_{i}, \sigma^{n}\right)$ 
can be treated as the distance between $c\left(\sigma^{n}\right)$ and $c\left(\sigma_{i}^{n-1}\right)$, and its sign can be computed by testing whether $c\left(\sigma^{n}\right)$ and $v_{i}$ have the same orientation with respect to aff $\left(\sigma_{i}^{n-1}\right)$ or not. Now by BDAMe, the heptahedron is the sum of the maximum number of six 3 -simplexes. Here we divide the quantity $h\left(v_{i}, \sigma^{n}\right)$ by the circumradius $R\left(\sigma^{n}\right)$ to get a quantity called cost function or energy function. Observe that, $-1<h\left(v_{i}, \sigma^{n}\right) / R\left(\sigma^{n}\right)<1$ for finite $\sigma^{n}$, as $R\left(\sigma^{n}\right)^{2}=h\left(v_{i}, \sigma^{n}\right)^{2}+R\left(\sigma_{i}^{n-1}\right)^{2}$. Then, we have the following theorem.

Theorem 2.1 The energy function (using BDAMe) $f_{p}\left(\sigma^{n}\right)=\frac{1}{N} \sum_{j=1}^{N} \max _{v \in \sigma^{n}}\left|\frac{h\left(v, \sigma_{j}^{n}\right)}{R\left(\sigma_{j}^{n}\right)}\right|$, where $N$ is number of tetrahedrons obtained using BDAMe of a 3D-figure (pentahedron, hexahedron, decahedron, octahedron, ..., etc), always lies between 0 and 1 that is, $0<$ $f_{p}\left(\sigma^{n}\right)<1$.

The proof of this theorem was already done in [16]. So, for our case, the energy function of heptahedron be $f_{H}\left(\sigma^{n}\right)=\frac{1}{6} \sum_{j=1}^{6} \max _{v \in \sigma^{n}}\left|\frac{h\left(v, \sigma_{j}^{n}\right)}{R\left(\sigma_{j}^{n}\right)}\right|$, where $\mathrm{H}$ stands for heptahedron.

\section{Methods of transformation}

In this section we use several methods of transformation to regularize the 3-D figure, like heptahedra. Here, we define a new apex transformation where the base points of the heptahedron are fixed.

\subsection{Transformation of heptahedra using GETMe:}

Let $H:=\left(h_{1}, h_{2}, h_{3}, h_{4}, h_{5}, h_{6}, h_{7}\right)^{t}$ denote a heptahedron with seven pairwise disjoint nodes $h_{i} \in R^{3}, i \in\{1, \ldots, 7\}$, which are positively oriented. Let

$$
\begin{aligned}
& n_{1}:=\left(h_{7}-h_{2}\right) \times\left(h_{3}-h_{2}\right), \\
& n_{2}:=\left(h_{7}-h_{3}\right) \times\left(h_{4}-h_{3}\right), \\
& n_{3}:=\left(h_{7}-h_{4}\right) \times\left(h_{5}-h_{4}\right), \\
& n_{4}:=\left(h_{7}-h_{5}\right) \times\left(h_{6}-h_{5}\right), \\
& n_{5}:=\left(h_{6}-h_{7}\right) \times\left(h_{1}-h_{7}\right), \\
& n_{6}:=\left(h_{1}-h_{7}\right) \times\left(h_{2}-h_{7}\right), \\
& n_{7}:=\left(h_{5}-h_{6}\right) \times\left(h_{1}-h_{6}\right),
\end{aligned}
$$

denote the inside oriented face normal of $\mathrm{H}$. A new heptahedron $H^{\prime}$ with nodes $h_{i}^{\prime}$ is derived from $H$ by constructing on each node $h_{i}$ the opposing face normal $n_{i}$ scaled by $\sigma / \sqrt{\left|n_{i}\right|}$, where $\sigma \in R_{0}^{+}$. That is 


$$
H^{\prime}=\left(\begin{array}{c}
h_{1}^{\prime} \\
h_{2}^{\prime} \\
h_{3}^{\prime} \\
h_{4}^{\prime} \\
h_{5}^{\prime} \\
h_{6}^{\prime} \\
h_{7}^{\prime}
\end{array}\right):=\left(\begin{array}{c}
h_{1} \\
h_{2} \\
h_{3} \\
h_{4} \\
h_{5} \\
h_{6} \\
h_{7}
\end{array}\right)+\sigma\left(\begin{array}{c}
\frac{1}{\sqrt{\left|n_{1}\right|}} n_{1} \\
\frac{1}{\sqrt{\left|n_{2}\right|}} n_{2} \\
\frac{1}{\sqrt{\left|n_{3}\right|}} n_{3} \\
\frac{1}{\sqrt{\left|n_{4}\right|}} n_{4} \\
\frac{1}{\sqrt{\left|n_{5}\right|}} n_{5} \\
\frac{1}{\sqrt{\left|n_{6}\right|}} n_{6} \\
\frac{1}{\sqrt{\left|n_{7}\right|}} n_{7}
\end{array}\right)
$$

It is clear that if $\sigma=0$ then $H^{\prime}$ and $H$ are same.

\subsection{Apex transformation of a heptahedron using GETMe:}

Apex transformation means, we transform the apex (top vertex) of the heptahedron using geometric element transformation method (GETMe) as discussed in the article (3.1). So, we transform $h_{7}$ (apex) to $h_{7}^{\prime}$ using only the inside oriented face normal $n_{7}$, $n_{7}:=\left(h_{5}-h_{6}\right) \times\left(h_{1}-h_{6}\right)$ of $H$. In that case, a new heptahedron $H^{\prime}$ with nodes $h_{i}^{\prime}$ is derived from $H$ by constructing the node $h_{7}$, the opposite face normal $n_{7}$ scaled by $\sigma / \sqrt{\left|n_{7}\right|}$, where $\sigma \in R_{0}^{+}$. So, we have given the apex transformation of heptahedron as below

$$
H^{\prime}=\left(\begin{array}{c}
h_{1}^{\prime} \\
h_{2}^{\prime} \\
h_{3}^{\prime} \\
h_{4}^{\prime} \\
h_{5}^{\prime} \\
h_{6}^{\prime} \\
h_{7}^{\prime}
\end{array}\right):=\left(\begin{array}{c}
h_{1} \\
h_{2} \\
h_{3} \\
h_{4} \\
h_{5} \\
h_{6} \\
h_{7}
\end{array}\right)+\sigma\left(\begin{array}{c}
0 \\
0 \\
0 \\
0 \\
0 \\
0 \\
\frac{1}{\sqrt{\left|n_{7}\right|}} n_{7}
\end{array}\right)
$$

It is clear that if $\sigma=0$ then $H^{\prime}$ and $H$ are same.

\subsection{New heptahedron derived from centroid transformation of a heptahe- dron using GETMe:}

Let $\mathrm{H}$ denotes a heptahedron with nodes $h_{k}$ and $\sigma \in R^{+}$an arbitary scaling factor. The nodes $h_{k}^{\prime}$ of the transformed heptahedron $H^{\prime}$ are given by

$$
h_{k}^{\prime}:=c_{k}+\frac{\sigma}{\sqrt{\left|n_{k}\right|}} n_{k}, k \in\{1, \ldots ., 7\} .
$$

that is $h_{k}^{\prime}$ is obtained by adding the centroid $c_{k}$ of the heptahedron face with the associated normal $n_{k}$ scaled by $\sigma / \sqrt{\left|n_{k}\right|}$. 


\subsection{Apex transformation of the heptahedron using centroid transformation:}

In this case we only transform the apex (top vertex) of the heptahedron using method (3). So, here we transform $h_{7}$ to $h_{7}^{\prime}$ and the transformed heptahedron is given by

$$
H^{\prime}=\left(\begin{array}{c}
h_{1}^{\prime} \\
h_{2}^{\prime} \\
h_{3}^{\prime} \\
h_{4}^{\prime} \\
h_{5}^{\prime} \\
h_{6}^{\prime} \\
h_{7}^{\prime}
\end{array}\right):=\left(\begin{array}{c}
c_{1} \\
c_{2} \\
c_{3} \\
c_{4} \\
c_{5} \\
c_{6} \\
c_{7}
\end{array}\right)+\sigma\left(\begin{array}{c}
0 \\
0 \\
0 \\
0 \\
0 \\
0 \\
\frac{1}{\sqrt{\left|n_{7}\right|}} n_{7}
\end{array}\right)
$$

here $c_{k}$ is the centroid of $k$ th heptahedron face where the associated normal $n_{k}$ scaled by $\sigma / \sqrt{\left|n_{k}\right|}, k \in\{1, \ldots . .7\}$ and $n_{7}:=\left(h_{5}-h_{6}\right) \times\left(h_{1}-h_{6}\right)$ of $H$.

\subsection{Apex transformation of the heptahedron using centroid transformation} but base points are fixed:

In this case we transform the apex of the heptahedron using centroid transformation but other base points are unaltered. So, here only transform $h_{7}$ to $h_{7}^{\prime}$ and we define the heptahedron is given by

$$
H^{\prime}=\left(\begin{array}{c}
h_{1}^{\prime} \\
h_{2}^{\prime} \\
h_{3}^{\prime} \\
h_{4}^{\prime} \\
h_{5}^{\prime} \\
h_{6}^{\prime} \\
h_{7}^{\prime}
\end{array}\right):=\left(\begin{array}{c}
h_{1} \\
h_{2} \\
h_{3} \\
h_{4} \\
h_{5} \\
h_{6} \\
c_{7}
\end{array}\right)+\sigma\left(\begin{array}{c}
0 \\
0 \\
0 \\
0 \\
0 \\
0 \\
\frac{1}{\sqrt{\left|n_{7}\right|}} n_{7} .
\end{array}\right)
$$


In the following figure we want to demonstrate the method (5)

$\mathrm{h}_{1}$

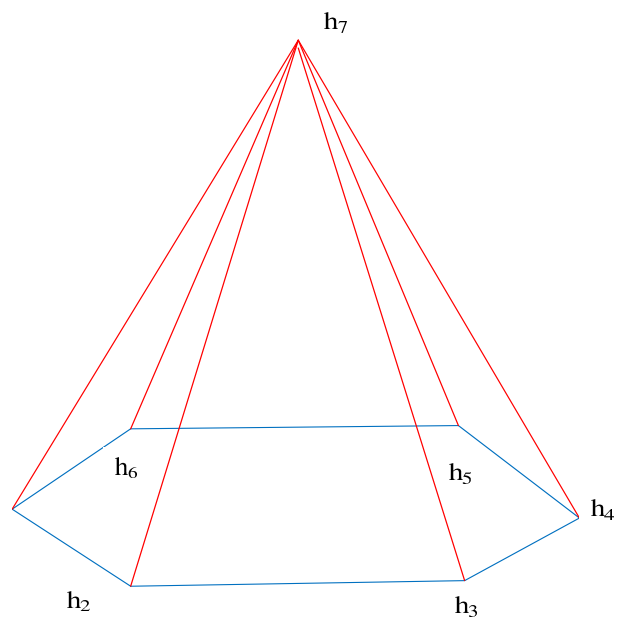

Fig.1: Before transformation of heptahedron

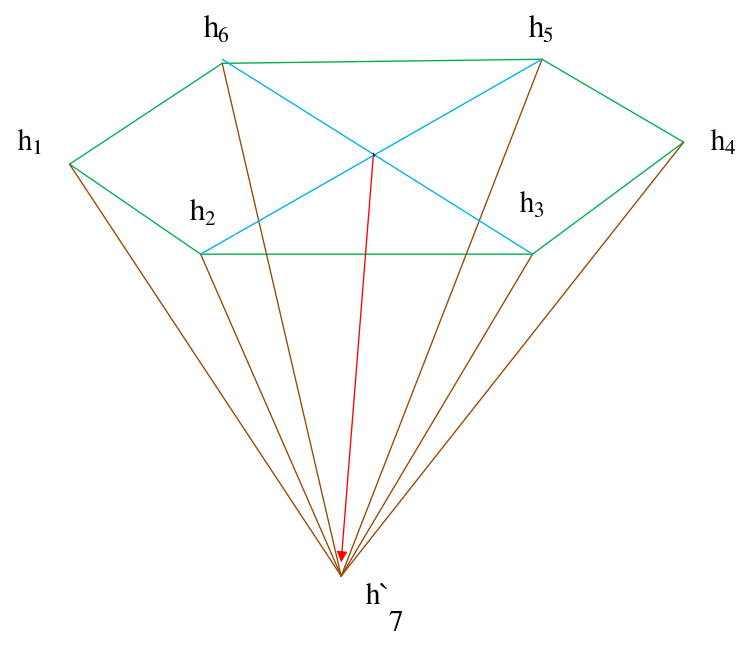

Fig.2: Deformed Heptahedron

\section{Properties of transformations:}

In this section, we discuss the basic properties of the above four transformations.

\subsection{The transformations are scale invariant:}

Proof: Since the normals $n_{i}$ are scaled by $1 / \sqrt{\left|n_{i}\right|}$, therefore the transformations given by (1), (2), (3), (4), (5) are scale invariant, that means for any $s>0,(s H)^{\prime}=s H^{\prime}$.

To check this property we shall consider some examples where we choose $\sigma=0.1$.

Example 1. In this example, we use the transformations (1) and then investigate the properties of $(s H)^{\prime}=s H^{\prime}$. Let $H:=\left(h_{1}, h_{2}, h_{3}, h_{4}, h_{5}, h_{6}, h_{7}\right)^{t}$ denote the heptahedron with $h_{1} \equiv(-.64, .48, .6), h_{2} \equiv(.8,0, .6), h_{3} \equiv(0,-.8, .6), h_{4} \equiv(-.8,0, .6), h_{5} \equiv(0, .8, .6)$, $h_{6} \equiv(.64,-.48, .6), h_{7} \equiv(0,0,1)$ Let $s=0.5$ and applying the transformation (1) both on $(s H)^{\prime}$ and $s H^{\prime}$ after that we get the following table 


\begin{tabular}{|c|c|c|c|}
\hline \multirow{2}{*}{} & \multicolumn{3}{|c|}{ Vertex Coordinates of $(s H)^{\prime}$} \\
\cline { 2 - 4 } & $\mathrm{x}$ & $\mathrm{y}$ & $\mathrm{z}$ \\
\hline$\left(s h_{1}\right)^{\prime}$ & -.3 & 0.22 & .34 \\
\hline$\left(s h_{2}\right)^{\prime}$ & .38 & -.02 & .34 \\
\hline$\left(s h_{3}\right)^{\prime}$ & .02 & -.38 & .34 \\
\hline$\left(s h_{4}\right)^{\prime}$ & -.37 & .02 & .33 \\
\hline$\left(s h_{5}\right)^{\prime}$ & .03 & .44 & .30 \\
\hline$\left(s h_{6}\right)^{\prime}$ & .31 & -.28 & .28 \\
\hline$\left(s h_{7}\right)^{\prime}$ & 0 & 0 & .55 \\
\hline
\end{tabular}

\begin{tabular}{|c|c|c|c|}
\hline \multirow{2}{*}{} & \multicolumn{3}{|c|}{ Vertex Coordinates of $s H^{\prime}$} \\
\cline { 2 - 4 } & $\mathrm{x}$ & $\mathrm{y}$ & $\mathrm{Z}$ \\
\hline$s h_{1}^{\prime}$ & -.3 & 0.22 & .34 \\
\hline$s h_{2}^{\prime}$ & .38 & -.02 & .34 \\
\hline$s h_{3}^{\prime}$ & .02 & -.38 & .34 \\
\hline$s h_{4}^{\prime}$ & -.37 & .02 & .33 \\
\hline$s h_{5}^{\prime}$ & .03 & .44 & .30 \\
\hline$s h_{6}^{\prime}$ & .31 & -.28 & .28 \\
\hline$s h_{7}^{\prime}$ & 0 & 0 & .55 \\
\hline
\end{tabular}

Hence for this heptahedron the transformation (1) is scale invariant. Similarly, we can show that the transformation (2) is also scale invariant.

Example 2. In this example, we use the transformation (3) and then try to investigate the property of $(s H)^{\prime}=s H^{\prime}$. We use the same heptahedron which is used in example (1) with $s=0.5$ and then applying the transformation (3) both on $(s H)^{\prime}$ and $s H^{\prime}$ and after calculations we get the following table

\begin{tabular}{|c|c|c|c|}
\hline \multirow{2}{*}{} & \multicolumn{3}{|c|}{ Vertex Coordinates of $(s H)^{\prime}$} \\
\cline { 2 - 4 } & $\mathrm{x}$ & $\mathrm{y}$ & $\mathrm{Z}$ \\
\hline$\left(s h_{1}\right)^{\prime}$ & .15 & -.15 & .40 \\
\hline$\left(s h_{2}\right)^{\prime}$ & -.15 & -.15 & .40 \\
\hline$\left(s h_{3}\right)^{\prime}$ & -.11 & .15 & .40 \\
\hline$\left(s h_{4}\right)^{\prime}$ & .13 & .07 & .39 \\
\hline$\left(s h_{5}\right)^{\prime}$ & .03 & .04 & .37 \\
\hline$\left(s h_{6}\right)^{\prime}$ & .01 & .40 & .36 \\
\hline$\left(s h_{7}\right)^{\prime}$ & 0 & .0 & .85 \\
\hline
\end{tabular}

\begin{tabular}{|c|c|c|c|}
\hline \multirow{2}{*}{} & \multicolumn{3}{|c|}{ Vertex Coordinates of $s H^{\prime}$} \\
\cline { 2 - 4 } & $\mathrm{x}$ & $\mathrm{y}$ & $\mathrm{z}$ \\
\hline$s h_{1}^{\prime}$ & .15 & -.15 & .40 \\
\hline$s h_{2}^{\prime}$ & -.15 & -.15 & .40 \\
\hline$s h_{3}^{\prime}$ & -.11 & .15 & .40 \\
\hline$s h_{4}^{\prime}$ & .13 & .07 & .39 \\
\hline$s h_{5}^{\prime}$ & .03 & .04 & .37 \\
\hline$s h_{6}^{\prime}$ & .01 & .40 & .36 \\
\hline$s h_{7}^{\prime}$ & 0 & .0 & .85 \\
\hline
\end{tabular}

Hence for this heptahedron the transformation (3) is scale invariant. One can also show that the transformation (4) is also scale invariant.

Example 3. In this example, we use the transformation (5) and then try to investigate the property of $(s H)^{\prime}=s H^{\prime}$. We use the same heptahedron which is used in example (1) with $s=0.5$ and then applying the transformation (5) both on $(s H)^{\prime}$ and $s H^{\prime}$ and after calculations we get the following table 


\begin{tabular}{|c|c|c|c|}
\hline \multirow{2}{*}{} & \multicolumn{3}{|c|}{ Vertex Coordinates of $(s H)^{\prime}$} \\
\cline { 2 - 4 } & $\mathrm{x}$ & $\mathrm{y}$ & $\mathrm{z}$ \\
\hline$\left(s h_{1}\right)^{\prime}$ & -.32 & 0.24 & .3 \\
\hline$\left(s h_{2}\right)^{\prime}$ & .4 & 0 & .3 \\
\hline$\left(s h_{3}\right)^{\prime}$ & 0 & -.4 & .3 \\
\hline$\left(s h_{4}\right)^{\prime}$ & -.4 & 0 & .3 \\
\hline$\left(s h_{5}\right)^{\prime}$ & 0 & .4 & .3 \\
\hline$\left(s h_{6}\right)^{\prime}$ & .32 & -.24 & .3 \\
\hline$\left(s h_{7}\right)^{\prime}$ & 0 & 0 & .85 \\
\hline
\end{tabular}

\begin{tabular}{|c|c|c|c|}
\hline \multirow{2}{*}{} & \multicolumn{3}{|c|}{ Vertex Coordinates of $s H^{\prime}$} \\
\cline { 2 - 4 } & $\mathrm{x}$ & $\mathrm{y}$ & $\mathrm{z}$ \\
\hline$s h_{1}^{\prime}$ & -.32 & 0.24 & .3 \\
\hline$s h_{2}^{\prime}$ & .4 & 0 & .3 \\
\hline$s h_{3}^{\prime}$ & 0 & -.4 & .3 \\
\hline$s h_{4}^{\prime}$ & -.4 & 0 & .3 \\
\hline$s h_{5}^{\prime}$ & 0 & .4 & .3 \\
\hline$s h_{6}^{\prime}$ & .32 & -.24 & .3 \\
\hline$s h_{7}^{\prime}$ & 0 & 0 & .85 \\
\hline
\end{tabular}

Hence for this heptahedron the transformation (5) is scale invariant.

4.2 Transformations (1), (2), (3), (4) and (5) do not preserve the centroid of the heptahedron:

Proof: The transformations given by (1), (2), (3), (4) and (5) do not preserve the centroid of the heptahedron, that is $\frac{1}{7} \sum_{i=1}^{7} h_{i} \neq \frac{1}{7} \sum_{i=1}^{7} h_{i}^{\prime}$, where $h_{1}, h_{2}, h_{3}, h_{4}, h_{5}, h_{6}$, $h_{7}$ are the vertex coordinates of original heptahedron and $h_{1}^{\prime}, h_{2}^{\prime}, h_{3}^{\prime}, h_{4}^{\prime}, h_{5}^{\prime}, h_{6}^{\prime}, h_{7}^{\prime}$ are the vertex coordinates of the transformed heptahedron. As the scale normals $n_{i} / \sqrt{\left|n_{i}\right|}$ have been used to ensure the scale invariance of the transformation, so the transformations (1), (2), (3), (4) and (5) do not preserve the centroid of the heptahedron.

We verify it by an examples.

Example 4. Let $\left(h_{1}, h_{2}, h_{3}, h_{4}, h_{5}, h_{6}, h_{7}\right)^{t}$ be call heptahedron where $h_{1} \equiv(1,0,1)$, $h_{2} \equiv(1,5,1), h_{3} \equiv(4,1,1), h_{4} \equiv(4,6,1), h_{5} \equiv(0,3,1), h_{6} \equiv(5,3,1), h_{7} \equiv(5,0,2)$ and after using transformation(1), we get $h_{1}^{\prime} \equiv(1.02, .06, .9), h_{2}^{\prime} \equiv(.9,5,1.1), h_{3}^{\prime} \equiv(4, .9, .9)$, $h_{4}^{\prime} \equiv(.4,6.03,1.1), h_{5}^{\prime} \equiv(-.02,3.03,1.1), h_{6}^{\prime} \equiv(5.02,3, .9), h_{7}^{\prime} \equiv(5,0,2.1)$

\begin{tabular}{|c|c|}
\hline \multicolumn{2}{|c|}{ Centroid of the heptahedron } \\
\hline Before transformation $\left(\frac{1}{7} \Sigma_{i=1}^{7} h_{i}\right)$ & After transformation $\left(\frac{1}{7} \Sigma_{i=1}^{7} h_{i}^{\prime}\right)$ \\
\hline$(2.8,2.6,1.1)$ & $(2.8,2.5,1.1)$ \\
\hline
\end{tabular}

Hence from the above we can say that the transformation (1) does not preserve the centroid of the transformation, provided after transformation the base of the heptahedron must also be coplanar. Similarly, we can also show that the transformation (2) does not preserve the centroid of transformation by the following table

\begin{tabular}{|c|c|}
\hline \multicolumn{2}{|c|}{ Centroid of the heptahedron } \\
\hline Before transformation $\left(\frac{1}{7} \Sigma_{i=1}^{7} h_{i}\right)$ & After transformation $\left(\frac{1}{7} \Sigma_{i=1}^{7} h_{i}^{\prime}\right)$ \\
\hline$(2.8,2.6,1.1)$ & $(2.8,2.6,1.2)$ \\
\hline
\end{tabular}


Example 5. In this example, we show that transformation (3) does not preserve centroid of transformation, provided after transformation the base of the heptahedron must also be coplanar. We take same co-ordinates of heptahedron as taken as example (1), we get the following table

\begin{tabular}{|c|c|}
\hline \multicolumn{2}{|c|}{ Centroid of the heptahedron } \\
\hline Before transformation $\left(\frac{1}{7} \Sigma_{i=1}^{7} h_{i}\right)$ & After transformation $\left(\frac{1}{7} \Sigma_{i=1}^{7} h_{i}^{\prime}\right)$ \\
\hline$(2.8,2.6,1.1)$ & $(3.2,2.2,1.2)$ \\
\hline
\end{tabular}

Similarly, we can also show that transformation (4) does not preserve the centroid of transformation.

Example 6. Here, we show that transformation (5) does not satisfy the preserving property of centroid of transformation. We take same co-ordinates of heptahedron as taken as example (1), we get the following table

\begin{tabular}{|c|c|}
\hline \multicolumn{2}{|c|}{ Centroid of the heptahedron } \\
\hline Before transformation $\left(\frac{1}{7} \Sigma_{i=1}^{7} h_{i}\right)$ & After transformation $\left(\frac{1}{7} \Sigma_{i=1}^{7} h_{i}^{\prime}\right)$ \\
\hline$(2.8,2.6,1.1)$ & $(2.5,3,1)$ \\
\hline
\end{tabular}

\subsection{Characterization of Mean Ratio Quality of a heptahedron:}

To define mean ratio quality for heptahedron, first we use BDAMe to get six tetrahedra and then choose any tetrahedron. Let $T:=\left(h_{1}, h_{2}, h_{3}, h_{4}\right)$ denote a tetrahedron with the four pairwise disjoint nodes $h_{i} \in R^{3}, i \in\{1, \ldots, 4\}$, which is positively oriented. That is $\operatorname{det}(A)>0$ with $A:=\left(h_{2}-h_{1}, h_{3}-h_{1}, h_{4}-h_{1}\right)$ representing the $(3 \times 3)$ Jacobian matrix of the difference vectors, which span the tetrahedron. In $[4,7,14-16]$, authors have discussed how to get mean ratio quality of a tetrahedron and using this procedure we define the mean ratio quality for heptahedron,

$$
q(H):=\frac{1}{6} \sum_{k=1}^{6} \frac{3 \operatorname{det}\left(S_{k}\right)^{2 / 3}}{\|S\|_{F}}
$$

, where $\left.\|S\|:=\operatorname{trace} \sqrt{(} S^{t} S\right)$ denote the Frobenious norm of the matrix $S_{k}=A_{k} W^{-1}$ and

$$
W=\left(\begin{array}{ccc}
1 & 1 / 2 & 1 / 2 \\
0 & \sqrt{3} / 2 & \sqrt{3} / 6 \\
0 & 0 & \sqrt{3} / \sqrt{2}
\end{array}\right)
$$

denotes the difference matrix of a regular reference tetrahedron. Now in the case of heptahedron, the criterion of $q(H)$ is not same as in $[15,16]$. In that case, if $H$ is regular then $q(H) \in[0,1]$, where very small values indicate nearly degenerated elements and large 
values element good quality. Now, if the transformation is applied iteratively, the resulting heptahedron became more and more regular. In order to assess the regularity of a heptahedron $H$ numerically, the mean ratio quality criterion will be used. Now, we give an example of a heptahedron which is regular base heptahedron but $q(H) \neq 1$.

Example 7. Let $\left(h_{1}, \ldots \ldots \ldots, h_{7}\right)^{t}$ be call heptahedron where $h_{1} \equiv(5,4,5), h_{2} \equiv$ $(5,9,5), h_{3} \equiv(0,9,5), h_{4} \equiv(0,4,5), h_{5} \equiv(3,8,5), h_{6} \equiv(8,8,5), h_{7} \equiv(4,9,11)$. Here, $q(H)=0.417$.

\subsection{Significance of the scaling factor $\sigma$ :}

$\sigma$ is playing a important role to regularize a heptahedron and the speed of the regularization. The resulting iteration numbers are totally depended upon the scaling factor $\sigma$.

Also the important fact is that there is no specific choice of $\sigma$, for which the transformation given exactly once to any arbitrary heptahedron results a regular one. To show this, we give an example.

Example 8. Let us choose the heptahedron with the same coordinate as given in article (4.2) example 1. According to (1), the nodes of the transformed heptahedron $H^{\prime}$ are given by $h_{1}^{\prime} \equiv(1+\sigma(.8), 0+\sigma(.6), 1+\sigma(-.2)), h_{2}^{\prime} \equiv(1+\sigma(-.7), 5+\sigma(0), 1+\sigma(.7)), h_{3}^{\prime} \equiv$ $(4+\sigma(.1), 1+\sigma(-.1), 1+\sigma(-.9)), h_{4}^{\prime} \equiv(4+\sigma(0), 6+\sigma(.3), 1+\sigma(.9)), h_{5}^{\prime} \equiv(0+\sigma(-.2), 3+$ $\sigma(.3), 1+\sigma(.9)), h_{6}^{\prime} \equiv(5+\sigma(.2), 3+\sigma(0), 1+\sigma(-.9))$ and $h_{7}^{\prime} \equiv(5+\sigma(0), 0+\sigma(0), 2+\sigma(1))$ using an arbitrary scaling factor $\sigma \in R_{0}^{+}$. In order to be regular, all the base lengths of the transformed heptahedron must be equal.

But, we see that $\left|h_{1}^{\prime}-h_{2}^{\prime}\right|=\left|h_{2}^{\prime}-h_{3}^{\prime}\right|$ for $\sigma=55.2,\left|h_{2}^{\prime}-h_{3}^{\prime}\right|=\left|h_{3}^{\prime}-h_{4}^{\prime}\right|$ for $\sigma=8$, $\left|h_{3}^{\prime}-h_{4}^{\prime}\right|=\left|h_{4}^{\prime}-h_{5}^{\prime}\right|$ for $\sigma=0.71,\left|h_{4}^{\prime}-h_{5}^{\prime}\right|=\left|h_{5}^{\prime}-h_{6}^{\prime}\right|$ for $\sigma=0.69,\left|h_{5}^{\prime}-h_{6}^{\prime}\right|=\left|h_{6}^{\prime}-h_{1}^{\prime}\right|$ for $\sigma=5.4$. So, we see that there is no specific $\sigma \in R_{0}^{+}$for which the heptahedron $H^{\prime}$ obtained by one step of the transformation is regular.

\subsection{Uniqueness of the circumsphere of heptahedron:}

Now for any 3-simplex, we can always draw a sphere through the four vertices of the 3-simplex, but for heptahedron and other 3D-figures we always do not get a sphere through the all vertices of the 3D-figure except tetrahedron. But if we choose a heptahedron so that its all vertices satisfy some particular sphere equation and then we use the transformation (1), (2), (3), (4) and (5), we can show that after transformation the transformed heptahedron may not satisfy some particular sphere equation. Let us give an example using transformation (2) and (4).

Example 9. Let $H:=\left(h_{1}, h_{2}, h_{3}, h_{4}, h_{5}, h_{6}, h_{7}\right)^{t}$ denote the heptahedron with $h_{1} \equiv$ $(-.64, .48, .6), h_{2} \equiv(.8,0, .6), h_{3} \equiv(0,-.8, .6), h_{4} \equiv(-.8,0, .6), h_{5} \equiv(0, .8, .6), h_{6} \equiv$ $(.64,-.48, .6), h_{7} \equiv(0,0,1)$. We see that all these points are satisfy the sphere equation 
$x^{2}+y^{2}+z^{2}=1$. But after transformation using (1), we see that they do not satisfy the sphere equation. If we use transformation (2), we get, $h_{1}^{\prime} \equiv(-.64, .48, .6), h_{2}^{\prime} \equiv(.8,0, .6)$, $h_{3}^{\prime} \equiv(0,-.8, .6), h_{4}^{\prime} \equiv(-.8,0, .6), h_{5}^{\prime} \equiv(0, .8, .6), h_{6}^{\prime} \equiv(.64,-.48, .6), h_{7}^{\prime} \equiv(0,0,1.1)$. We see that they also do not satisfy the sphere equation. One can show that transformation (3) does not satisfy the sphere equation. If we use apex transformation (4), we get the co-ordinates $h_{1}^{\prime} \equiv(.26,-.26, .73), h_{2}^{\prime} \equiv(-.26,-.26, .73), h_{3}^{\prime} \equiv(-.26, .26, .73)$, $h_{4}^{\prime} \equiv(.21, .11, .73), h_{5}^{\prime} \equiv(0,0, .73), h_{6}^{\prime} \equiv(.05, .16, .73), h_{7}^{\prime} \equiv(0,0, .73)$ We see that they also do not satisfy the sphere equation.

Example 10. If we see transformation (5), the transformed heptahedron becomes $h_{1} \equiv(-.64, .48, .6), h_{2} \equiv(.8,0, .6), h_{3} \equiv(0,-.8, .6), h_{4} \equiv(-.8,0, .6), h_{5} \equiv(0, .8, .6)$, $h_{6} \equiv(.64,-.48, .6), h_{7}^{\prime} \equiv(0,0,1.7)$. We see that all $h_{i}^{\prime}$ do not satisfy the sphere equation.

\section{Regularization of a heptahedron}

Here we shall consider a process to regularize a heptahedron. In the case of heptahedron regular means that the base of the heptahedron is regular and the upper all edge lengths are equal. So, when we consider an arbitrary heptahedron it is quite difficult to regularize the heptahedron.

Observation 5.1 If the base of the heptahedron is regular and upper portion of the heptahedron is irregular then we can regularize the heptahedron using any of the transformations (1), (2), (3), (4) and (5).

This can be verified by some examples where transformations (2), (4) and (5) are used to regularize the heptahedron whose base is regular.

Example 11. Let $H:=\left(h_{1}, h_{2}, h_{3}, h_{4}, h_{5}, h_{6}, h_{7}\right)^{t}$ denote a heptahedron with $h_{1} \equiv$ $(5,4,5), h_{2} \equiv(5,8,5), h_{3} \equiv(1,8,5), h_{4} \equiv(1,4,5), h_{5} \equiv(1,0,5), h_{6} \equiv(5,0,5)$ and $h_{7} \equiv(4,8,10)$. This heptahedron is not regular because the base edges length $h_{1} h_{2}=$ $h_{2} h_{3}=h_{3} h_{4}=h_{4} h_{5}=h_{5} h_{6}=h_{6} h_{1}=4$ and for the upper portion length of the edges are $h_{1} h_{7}=6.48, h_{2} h_{7}=5.09, h_{3} h_{7}=5.83, h_{4} h_{7}=7.07, h_{5} h_{7}=9.89, h_{6} h_{7}=9.48$ which all are not equal. Now, if we use the transformation (2) on the given heptahedron, then in first step, length of the edges (upper portion) are $h_{1}^{\prime} h_{7}^{\prime}=6.40, h_{2}^{\prime} h_{7}^{\prime}=5, h_{3}^{\prime} h_{7}^{\prime}=5.74$, $h_{4}^{\prime} h_{7}^{\prime}=7, h_{5}^{\prime} h_{7}^{\prime}=9.84, h_{6}^{\prime} h_{7}^{\prime}=9.43$ and in 2nd step, length of the edges are $h_{1}^{\prime \prime} h_{7}^{\prime \prime}=6.32$, $h_{2}^{\prime \prime} h_{7}^{\prime \prime}=4.9, h_{3}^{\prime \prime} h_{7}^{\prime \prime}=5.66, h_{4}^{\prime \prime} h_{7}^{\prime \prime}=6.93, h_{5}^{\prime \prime} h_{7}^{\prime \prime}=9.80, h_{6}^{\prime \prime} h_{7}^{\prime \prime}=9.38$. Here we observe that the heptahedron tends to regularize but slowly. The speed of the regularization depends upon the choice of the scaling factor $\sigma$. In this case we take the scaling factor $\sigma=0.1$.

If we use same points on the heptahedron using transformation (3), we get the length of the edges (upper portion) are $h_{1}^{\prime} h_{7}^{\prime}=4.3, h_{2}^{\prime} h_{7}^{\prime}=3.3, h_{3}^{\prime} h_{7}^{\prime}=1.9, h_{4}^{\prime} h_{7}^{\prime}=2.2, h_{5}^{\prime} h_{7}^{\prime}=2.5$, $h_{6}^{\prime} h_{7}^{\prime}=3.1$. So, we see that upper portion of this polyhedron is not regular. But, we look after transformation, the base points are regular i.e, $h_{1}^{\prime} h_{2}^{\prime}=h_{2}^{\prime} h_{3}^{\prime}=h_{3}^{\prime} h_{4}^{\prime}=h_{4}^{\prime} h_{5}^{\prime}=$ 
$h_{5}^{\prime} h_{6}^{\prime}=h_{6}^{\prime} h_{1}^{\prime}$. But, if we use the transformation (5) after the 1st step of transformation (3) on heptahedron, we get, $h_{1}^{\prime \prime}=h_{1}^{\prime}, h_{2}^{\prime \prime}=h_{2}^{\prime}, h_{3}^{\prime \prime}=h_{3}^{\prime}, h_{4}^{\prime \prime}=h_{4}^{\prime}, h_{5}^{\prime \prime}=h_{5}^{\prime}, h_{6}^{\prime \prime}=h_{6}^{\prime}$ and $h_{7}^{\prime \prime}=(3.45,5.3,6.5)$ and we also see that the length of the edges of the upper portion of the heptahedron becomes $h_{1}^{\prime \prime} h_{7}^{\prime \prime}=2.6, h_{2}^{\prime \prime} h_{7}^{\prime \prime}=2, h_{3}^{\prime \prime} h_{7}^{\prime \prime}=2, h_{4}^{\prime \prime} h_{7}^{\prime \prime}=2.6, h_{5}^{\prime \prime} h_{7}^{\prime \prime}=2$, $h_{6}^{\prime \prime} h_{7}^{\prime \prime}=2.2$. So, we observe that the given heptahedron converges to regularize and it becomes a regular heptahedron (approximately).

Now, if we use the transformation (4), we get $h_{7}^{\prime}=(3,4,4.9)$. And after the first step, we see that length of the edges (upper portion) of the given heptahedron is not equal. If we use this transformation after second step, we get $h_{7}^{\prime \prime}=(3.3 .5 .3,6.6)$ and we observe also that the length of the upper portion is not regular. But after third step, we get $h_{7}^{\prime \prime \prime}=(3.2,4.9,6)$ and we see that $h_{1}^{\prime \prime \prime} h_{7}^{\prime \prime \prime}=.8, h_{2}^{\prime \prime \prime} h_{7}^{\prime \prime \prime}=.7, h_{3}^{\prime \prime \prime} h_{7}^{\prime \prime \prime}=.8, h_{4}^{\prime \prime \prime} h_{7}^{\prime \prime \prime}=1$, $h_{5}^{\prime \prime \prime} h_{7}^{\prime \prime \prime}=.8, h_{6}^{\prime \prime \prime} h_{7}^{\prime \prime \prime}=.6$. So, we see that it tends to regularize. So the deformed heptahedron becomes a regular heptahedron.

If we apply transformation (5) after first transformation of (4) on heptahedron, we get $h_{1}^{\prime \prime}=c_{1}, h_{2}^{\prime \prime}=c_{2}, h_{3}^{\prime \prime}=c_{3}, h_{4}^{\prime \prime}=c_{4}, h_{5}^{\prime \prime}=c_{5}, h_{6}^{\prime \prime}=c_{6}$ and $h_{7}^{\prime \prime}=(3.3,5.3,6.6)$. We observe that the length of the upper portion $h_{1}^{\prime \prime} h_{7}^{\prime \prime}=2.7, h_{2}^{\prime \prime} h_{7}^{\prime \prime}=2, h_{3}^{\prime \prime} h_{7}^{\prime \prime}=2, h_{4}^{\prime \prime} h_{7}^{\prime \prime}=2.7$, $h_{5}^{\prime \prime} h_{7}^{\prime \prime}=2, h_{6}^{\prime \prime} h_{7}^{\prime \prime}=2$. So, it also tends to regularize (approximately). All the above case the scaling factor $\sigma=.1$.

Example 12. Now, we use transformation (5) on the example $h_{1} \equiv(1,0,1), h_{2} \equiv$ $(1,5,1), h_{3} \equiv(4,1,1), h_{4} \equiv(4,6,1), h_{5} \equiv(0,3,1), h_{6} \equiv(5,3,1), h_{7} \equiv(5,0,2)$, the transformed heptahedron becomes $h_{1}^{\prime} \equiv(1,0,1), h_{2}^{\prime} \equiv(1,5,1), h_{3}^{\prime} \equiv(4,1,1), h_{4}^{\prime} \equiv(4,6,1)$, $h_{5}^{\prime} \equiv(0,3,1), h_{6}^{\prime} \equiv(5,3,1)$ and $h_{7}^{\prime} \equiv(2.5,3,1.1)$ taking scaling factor $\sigma=.1$ and the length of the upper portion are $h_{1}^{\prime} h_{7}^{\prime}=3.3, h_{2}^{\prime} h_{7}^{\prime}=2.5, h_{3}^{\prime} h_{7}^{\prime}=2.5, h_{4}^{\prime} h_{7}^{\prime}=3.3, h_{5}^{\prime} h_{7}^{\prime}=2.5$, $h_{6}^{\prime} h_{7}^{\prime}=2.5$. From this it is clear that this transformed heptahedron converges to regular (approx).

From the above examples we observe that the transformation (5) plays an important role to regularize the heptahedron.

\section{Characterization of energy function of a particular type of heptahedron using GETMe and BDAMe}

The changing cost function, after transforming the heptahedron by GETMe, is given by

$$
f\left(\sigma_{H}\right)=\left|f_{H_{k}}\left(\sigma^{n}\right) \sim f_{H_{k+1}}\left(\sigma^{n}\right)\right|
$$

Now using BDAMe we find the numerical values of changing cost function. We can calculate the changing cost function of the heptahedron provided after transformation the base points are coplanar. Let us consider an example where $h_{1} \equiv(5,2,5), h_{2} \equiv(5,7,5)$, $h_{3} \equiv(0,7,5), h_{4} \equiv(4,4,5), h_{5} \equiv(0,1,5), h_{6} \equiv(5,1,5)$ are the base points and $h_{7} \equiv(4,5,8)$ denote the apex of the the heptahedron. Here, $(2.5,4,5)$ be the inter- 
secting point of the base diagonals of the square part of the heptahedron. It has shown in the following figure

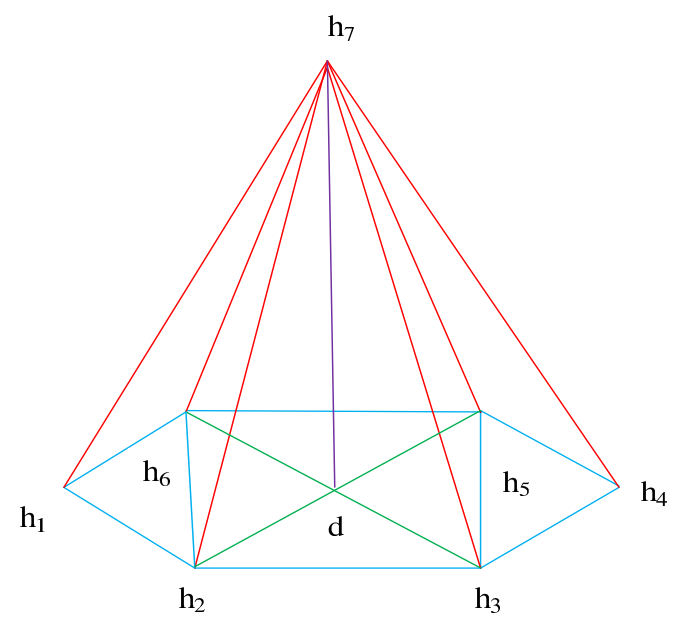

Fig.3: Characterization of heptahedron using BDAMe.

After calculation, we get

\begin{tabular}{|c|c|c|c|c|}
\hline \multirow{2}{*}{} & \multicolumn{4}{|c|}{ Apex Transformations } \\
\cline { 2 - 5 } & Initial Step & 2nd Step & 3rd Step & 4th Step \\
\hline$f_{H_{k}}\left(\sigma^{n}\right)$ & 0.6999 & 0.7251 & 0.7507 & 0.7770 \\
\hline
\end{tabular}

By this example we can show that the heptahedron converges to regularize on the transformation (2). We see in Figure (4) that the values of the changing cost function $f\left(\sigma_{H}\right)$ are $0.0252,0.0257$ and 0.0263 . 


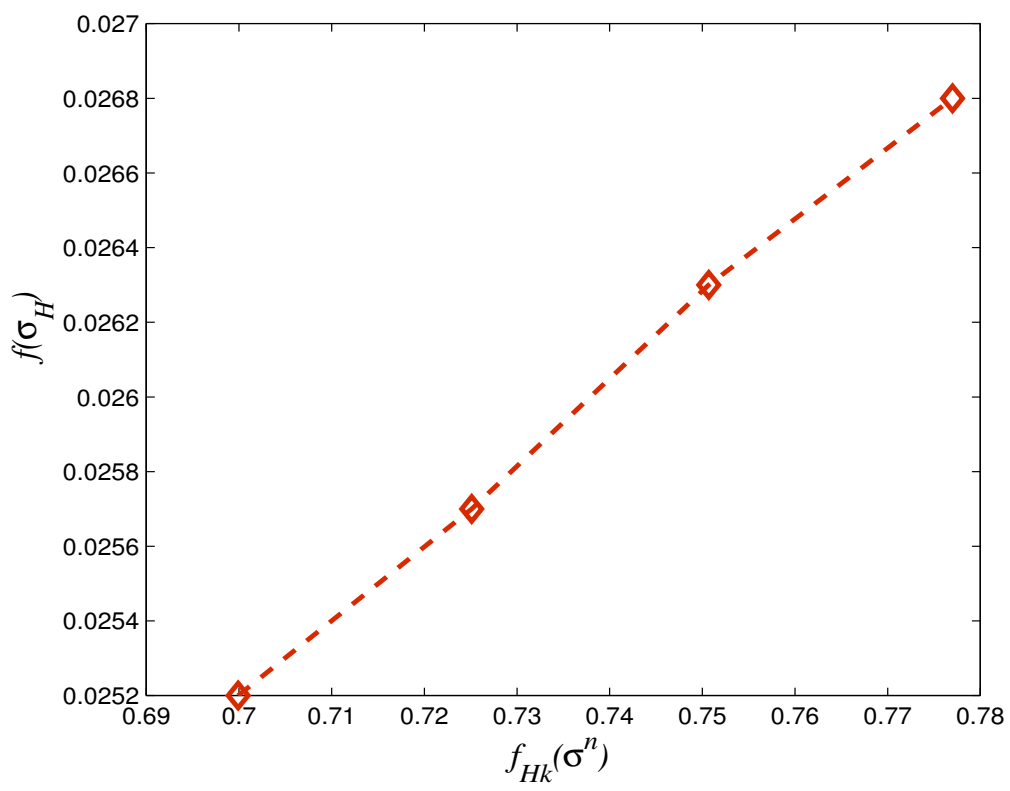

Fig.4: Changing cost function.

Therefore for this example we see that when it converges to regularize, the changing cost function also increases. One can also calculate the changing cost function for any arbitrary heptahedron (using the method (1) and (3)) provided after transformations the base points are coplanar.

\section{Conclusion}

One can regularize any 3-D figures, which are not simplex, using GETMe and BDAMe.

\section{Acknowledgements}

The authors wish to express their sincere thanks and gratitude to the referee for valuable suggestions towards the improvement of the paper. The first author is supported by DST/INSPIRE Fellowship/2013/1041, Govt. of India.

\section{References}

[1] Arindam Bhattacharyya and Buddhadev Pal. Quality statistics of octahedron and decahedron with the help of c-programs. Int. J. Pure Appl. Sci. Technol, 1(2):26-41, 2010 .

[2] JM Escobar, E Rodrıguez, R Montenegro, G Montero, and JM González-Yuste. Si- 
multaneous untangling and smoothing of tetrahedral meshes. Computer Methods in Applied Mechanics and Engineering, 192(25):2775-2787, 2003.

[3] Lori A Freitag and Patrick M Knupp. Tetrahedral element shape optimization via the jacobian determinant and condition number. In IMR, pages 247-258, 1999.

[4] Lori A Freitag and Patrick M Knupp. Tetrahedral mesh improvement via optimization of the element condition number. International Journal for Numerical Methods in Engineering, 53(6):1377-1391, 2002.

[5] Lori A Freitag, Paul Plassmann, et al. Local optimization-based simplicial mesh untangling and improvement. International Journal for Numerical Methods in Engineering, 49(1):109-125, 2000.

[6] Patrick Knupp. Remarks on mesh quality. Technical report, Sandia National Laboratories (SNL-NM), Albuquerque, NM (United States), 2007.

[7] Patrick M Knupp. Algebraic mesh quality metrics. SIAM journal on scientific computing, 23(1):193-218, 2001.

[8] Buddhadev Pal and Arindam Bhattacharyya. Regularization and energy estimation of pentahedra (pyramids) using geometric element transformation method. Mathematical Combinatorics, 1:67-79, 2014.

[9] Jonathan Shewchuk. What is a good linear finite element? interpolation, conditioning, anisotropy, and quality measures (preprint). University of California at Berkeley, 73, 2002 .

[10] Evan Vanderzee, Anil N Hirani, Damrong Guoy, and Edgar A Ramos. Well-centered triangulation. SIAM Journal on Scientific Computing, 31(6):4497-4523, 2010.

[11] Dimitris Vartziotis, Theodoros Athanasiadis, Iraklis Goudas, and Joachim Wipper. Mesh smoothing using the geometric element transformation method. Computer Methods in Applied Mechanics and Engineering, 197(45):3760-3767, 2008.

[12] Dimitris Vartziotis and Joachim Wipper. Classification of symmetry generating polygontransformations and geometric prime algorithms. Math. Pannonica, 20(2):167$187,2009$.

[13] Dimitris Vartziotis and Joachim Wipper. The geometric element transformation method for mixed mesh smoothing. Engineering with Computers, 25(3):287-301, 2009 .

[14] Dimitris Vartziotis and Joachim Wipper. Characteristic parameter sets and limits of circulant hermitian polygon transformations. Linear Algebra and its Applications, 433(5):945-955, 2010. 
[15] Dimitris Vartziotis and Joachim Wipper. A dual element based geometric element transformation method for all-hexahedral mesh smoothing. Computer Methods in Applied Mechanics and Engineering, 200(9):1186-1203, 2011.

[16] Dimitris Vartziotis, Joachim Wipper, and Bernd Schwald. The geometric element transformation method for tetrahedral mesh smoothing. Computer Methods in Applied Mechanics and Engineering, 199(1):169-182, 2009. 